\title{
Energy Efficient Algorithm with Considering Port- Cost in Hybrid Hierarchical Optical Networks
}

\author{
Weigang Hou, Lei Guo, Xingwei Wang \\ College of Information Science and Engineering, Northeastern University, Shenyang 110004, China
}

\begin{abstract}
In recent years, as the increasing effect of greenhouse and extremely consumption of worldwide energy, port-cost and energy-consumption savings are becoming a dual of new objectives of further developing an optical network. Using hybrid technology to replace Optical-Electrical-Optical (OEO) fabric with an all optical (OOO) one to save more consumed energy and using hierarchical technology to aggregate several wavelengths into wavebands to perform port-cost savings is an attractive solution for next-generation optical networks. In this paper, we jointly consider port-cost and energy-consumption in hybrid hierarchical optical network and develop efficient approaches ranging from Integer Linear Programming (ILP) models to heuristics. Simulation results of two heuristic approaches show that, the cost of Transmitting Ports (TPs) used in 000 switch is contrary to the energy consumed by OEO part of hybrid hierarchical Optical Cross Connect (OXC) node, and it is necessary to make a new design to obtain satisfactory trade-off performance.
\end{abstract}

Key words-hybrid hierarchical optical network, energyconsumption, port-cost, heuristic

\section{INTRODUCTION}

Using wavelength routing by OXCs has been the most feasible solution for optical network. However, the benefits of this technology come at the expense of increased number of wavelengths. Large numbers of available wavelengths result in increased port-cost of OXCs and increased difficulty in maintaining OXCs [1,2]. On the other hand, as the increasing effect of greenhouse and extremely consumption of worldwide energy, energy-consumption has been a new bottleneck of developing future network [3-5]. Therefore, port-cost and energy-consumption should be jointly considered in optical networks, where the port-cost mainly comes from the Transmitting Ports (TPs) used to switch through traffic, while the energy-consumption mainly comes from OEO conversions for processing individual wavelengths. Hybrid hierarchical technology proposed in Refs $[6,7]$ provided an attractive solution for minimizing port-cost and energy-consumption in the next-generation optical networks. This is because that using hybrid technology to replace OEO fabric with an $\mathrm{OOO}$ one can save more energy-consumption and using hierarchical technology to aggregate several wavelengths into wavebands followed by some waveband merging strategies can save more TPs.

On the node level, the node supporting hybrid hierarchical technology had been described in Refs $[6,7]$. This kind of node consists of $\mathrm{OOO}$ and $\mathrm{OEO}$ switches. The $\mathrm{OOO}$ switch is used to route through traffic while the OEO switch is used to add/drop local traffic or perform wavelength conversions. On the other hand, some waveband merging strategies had also been proposed in Refs [6-9]. Among which, in Ref [7], based on hybrid hierarchical OXC node, the authors proposed two waveband routing and merging strategies, Full OEO-based Merging (FEM) and Partial OEO-based Merging (PEM). Simulation results showed that the proposed algorithms provided higher cost benefit compared to existing algorithms without waveband merging architecture. An analytical model was proposed in Ref [8] to determine the network blocking probability for one waveband merging strategy called samedestination-intermediate, and the results indicated that, this strategy outperforms End-to-End waveband Merging strategy (EEM) in terms of port-cost savings. The Sub Path Merging strategy (SPM) considered in Ref [9] is the most powerful in terms of maximizing the benefits of WaveBand Switch (WBS) by increasing the grouping of wavelengths, although it is also the most complex to use in WBS algorithms.

However, the researches mentioned above, especially for WBS algorithms and waveband merging strategies used in hybrid hierarchical optical network, only concentrate on portcost savings, the issue of energy-consumption savings is not considered in those researches.

Therefore, in this paper, we propose a new routing method based on hybrid hierarchical OXC node, develop a port-cost and energy-consumption minimization model for hybrid hierarchical optical network and compare the performance of port-cost and energy-consumption savings for two heuristic approaches: Cost and Energy savings algorithm based on EEM (CEEEM) as well as Cost and Energy savings algorithm based on SPM (CESPM). To the best of our knowledge, the work in this paper is the first study to jointly consider portcost and energy-consumption.

The rest of this paper is organized in the following manner: Section 2 describes the structure of hybrid hierarchical OXC node. Section 3 states an Integer Linear Programming (ILP) model with the objective to minimize the port-cost and energy-consumption in hybrid hierarchical optical network. Two heuristic approaches, CEEEM and CESPM, are presented in Section4. Section 5 is for simulation and analysis. Section 6 concludes this paper.

\section{HYBRID HIERARCHICAL OXC NODE}




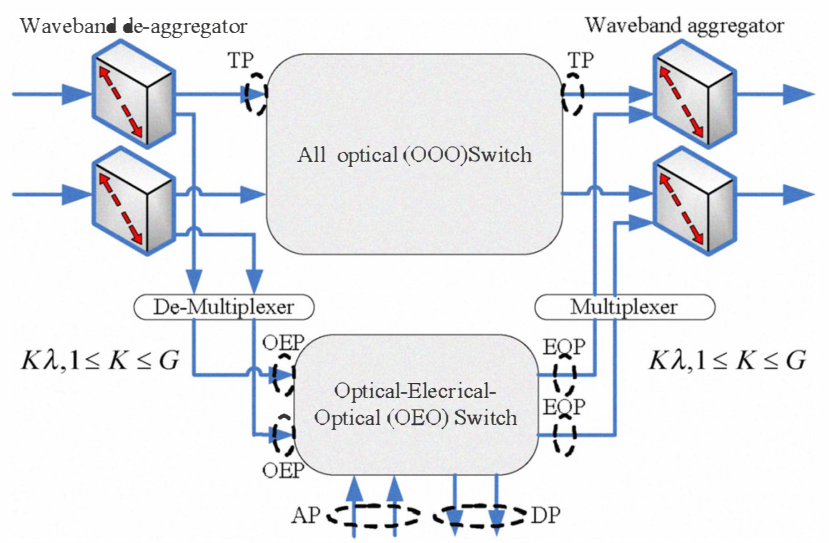

Fig.1 Hybrid hierarchical OXC node

The node in the hybrid hierarchical optical network consists of an $\mathrm{OOO}$ switch and an OEO switch, as shown in Fig.1. When a waveband is switched in OOO domain, only two TPs are used at each bypass node. Once an individual wavelength can not be merged into waveband, that wavelength will be blocked. Therefore, compared to switching them in OEO domain, this routing method can further save energy in OEO part of the node. However, in order to obtain lower blocking probability, using a rational waveband merging strategy to group as many wavelengths as possible into wavebands is also important. Transferring one request from $\mathrm{OOO}$ switch to OEO switch or in an inverse way can be performed by using Optical-to-Electrical Port (OEP) and Electrical-to-Optical Port (EOP), respectively. The Add Port (AP) is designed to add one request locally while the Drop Port (DP) is designed to drop one request locally. If the request uses multiple waveband-paths, wavelength conversions are needed in OEO part of the node when the request is switched from one waveband to another different one.

\section{PORT-COST AND ENERGY-CONSUMPITON MINIMIZAITON MODEL}

\section{A. Notations}

$V:$ Set of network nodes;

$(n, m)$ : Link between node $n(n \in V)$ and node $m(m \in V)$;

$\mathrm{A}(n)$ : Set of adjacent nodes to node $n(n \in V)$;

$I T P_{o o o}^{n}$ : Set of input TPs in OOO switch at node $n$ where incoming links $(m, n), m \in \mathrm{A}(n)$ are terminated;

OTP ${ }_{o o o}^{n}$ : Set of output TPs in OOO switch at node $n$ where outgoing links $(n, m), m \in \mathrm{A}(n)$ are originated;

$O E P_{o e o}^{n}$ : Set of input OEPs in OEO switch at node $n$ where incoming links $(m, n), m \in \mathrm{A}(n)$ are terminated;

$E O P_{o e o}^{n}$ : Set of output OEPs in OEO switch at node $n$ where outgoing links $(n, m), m \in \mathrm{A}(n)$ are originated;

$A L_{\text {oeo }}^{n}$ : Set of local add ports in OEO switch at node $n$;

$D L_{o e o}^{n}$ : Set of local drop ports in OEO switch at node $n$;
$I P L_{o o o}^{n}: A L_{o o o}^{n} \cup O E P_{o e o}^{n}$. Set of all input ports in OEO switch at node $n$;

$O P L_{o e o}^{n}: D L_{o e o}^{n} \cup E O P_{o o o}^{n}$. Set of all output ports in OEO switch at node $n$;

$P_{i}^{n}: I T P_{o o o}^{n} \cup I P L_{o e o}^{n}$. Set of all input ports at node $n$;

$P_{o}^{n}: O T P_{o o o}^{n} \cup O P L_{\text {oeo }}^{n}$. Set of all output ports at node $n$;

$Q$ : Number of available wavelengths in the whole network;

$\Delta$ : Set of available wavelengths in the whole network, $\Delta=\{0,1, \ldots . ., Q-1\}$;

$k, l, f:$ Wavelengths from set $\Delta$;

$G$ : Maximum number of contiguous wavelengths in one waveband;

$P$ : Number of available wavebands in the whole network;

$B$ : Set of available waveband in the whole network, $B=\{0,1, \ldots . . . P-1\}$;

$\Omega$ : Set of wavelengths that can be grouped into wavebands in the whole network,

$\Omega=\{\beta G, \beta G+1, \ldots \ldots,(\beta+1) G-1, \forall \beta=(0,1, \ldots \ldots, P-1) ;$

$T:$ Number of node pairs having non-zero traffic demand;

$C_{t}$ : Number of connection requests to be provisioned between the node pair $\left(s_{t}, d_{t}\right), \forall t=(0,1, \ldots \ldots . . T-1)$, where $s_{t}$ and $d_{t}$ represent the source and destination node, respectively; $R_{t}$ : Set of connection requests of size $C_{t}$ provisioned between the node pair $\left(s_{t}, d_{t}\right), R_{t}=\left\{r_{t}^{0}, r_{t}^{1}, \ldots . . . r_{t}^{C_{t}-1}\right\}$;

$R$ : Set of all connection requests of size $|R|=\sum_{t=0}^{T-1} C_{t}$ to be provisioned, $R=\left\{r_{t}^{j}: 0 \leq t \leq T, 0 \leq j \leq C_{t}\right\}$;

$|\phi|:$ Number of elements in set $\phi$.

\section{B. ILP variables}

$W_{r_{t}^{\prime}, i, o}^{n, k, l}: 1$ if at node $n$ a connection request $r_{t}^{j}\left(r_{t}^{j} \in R\right)$ uses wavelength $k(k \in \Omega)$ at input port $i\left(i \in I P L_{o o o}^{n}\right)$ in OEO switch and wavelength $l(l \in \Omega)$ at output port $o\left(o \in O P L_{o o o}^{n}\right)$ in OEO switch; otherwise it is 0 ;

$W B_{i, o}^{n, \beta}: 1$ if at node $n$ a waveband $\beta, \beta=(0,1, \ldots \ldots, P-1)$ from input port $i\left(i \in I T P_{o o o}^{n}\right)$ is switched through the $\mathrm{OOO}$ switch to output port $o\left(o \in O T P_{o o o}^{n}\right)$; otherwise, it is 0 ;

If a variable $W B_{i, o}^{n, \beta}$ is set to 1 , then all active wavelengths within the waveband $\beta$ are switched as a single entity through the $\mathrm{OOO}$ switch. The traffic at a node can be through traffic, add traffic, drop traffic or wavelength-convertible traffic.

The variable $W_{r_{l}^{\prime}, i, o}^{n, k}$ represents through traffic when $i \in O E P_{\text {oeo }}^{n}, o \in E O P_{o e o}^{n}, k=l$, add traffic when $i \in A L_{o e o}^{n}$, $o \in E O P_{o o o}^{n}, k=l$, drop traffic when $i \in O E P_{o e o}^{n}, o \in D L_{o o o}^{n}$, 


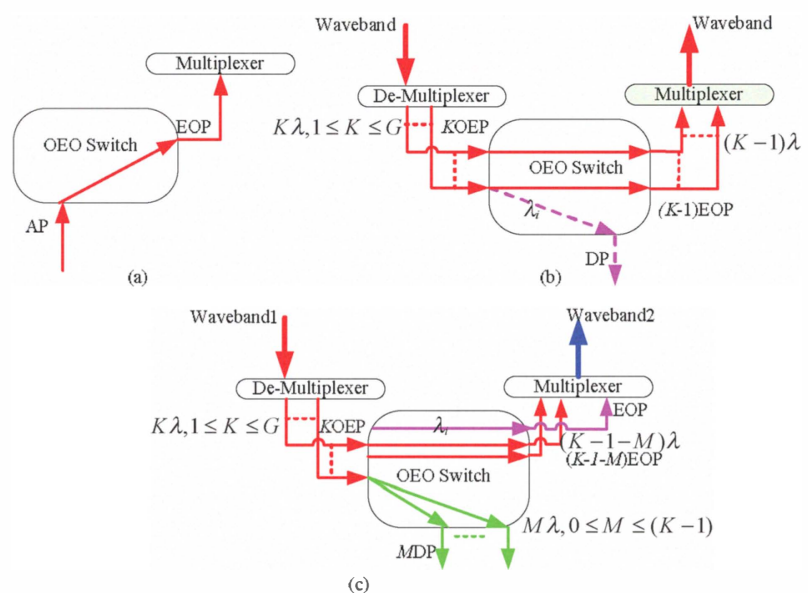

Fig.2 Energy consumption dynamically in OEO switch

$k=l$, or wavelength-convertible traffic when $i \in O E P_{o e o}^{n}$, $o \in E O P_{o e o}^{n}, k \neq l$.

\section{Minimizing the port-cost and energy-consumption}

Our objective is to minimize the port-cost of TPs in OOO switch and the energy-consumption of OEO ports in OEO switch. The objective function is defined as

$$
\Psi=\min \sum_{n \in V}\left[\alpha \eta_{n}+\gamma \delta_{n}\right],
$$

where

$$
\begin{aligned}
& \eta_{n}=\sum_{i \in I T P_{o o o}^{n}, o \in O T P_{o o o}^{n}, \beta \in B} W B_{i, o}^{n, \beta} \\
& \delta_{n}=\sum_{r_{t}^{\prime} \in R, i \in I P L_{0 e o, o \in O P L_{o e o}^{n}, k \in \Omega, l \in \Omega}^{n}} W_{r_{t}^{\prime}, i, o}^{n, k, l}
\end{aligned}
$$

subject to:

$$
\begin{aligned}
& \sum_{i \in A L_{0 e o, o \in O P L_{b e o}^{n}, k, l \in \Omega}^{n}} W_{r_{i}^{\prime}, i, o}^{n, k, l}=0, \quad n \neq s_{t}, n \neq d_{t} \\
& \sum_{i \in I P L_{o o o}^{n}, o \in D L_{0 e o, k, l \in \Omega}^{n}} W_{r_{t}^{\prime}, i, o}^{n, k, l}=0, \quad n \neq s_{t}, n \neq d_{t} \\
& \sum_{i \in O E P_{O e o}^{n}, o \in O P L_{D e o}^{n}, k, l \in \Omega} W_{r_{t}^{\prime}, i, o}^{n, k, l}=0, \quad n=s_{t}
\end{aligned}
$$

$$
\begin{aligned}
& \sum_{i \in A L_{0 e o, o \in E O P_{0 o O}^{n}, k, l \in \Omega}^{n}} W_{r_{t}^{\prime}, i, o}^{n, k, l}=1, \quad n=s_{t} \\
& \sum_{i \in I P L_{p e o, o}^{n}, o \in E O P_{0 \text { eo }, k, l \in \Omega}^{n},} W_{r_{t}^{\prime}, i, o}^{n, k, l}=0, \quad n=d_{t} \\
& \sum_{i \in O E P_{o e o, o \in D L}^{n} L_{o e o}, k, l \in \Omega} W_{r_{t}^{\prime}, i, o}^{n, k, l}=1, \quad n=d_{t} \\
& \sum_{r_{t}^{\prime} \in R, i \in A L_{o e o}^{n}, o \in D L_{0 e o, k, l \in \Omega}^{n}} W_{r_{t}^{\prime}, i, o}^{n, k, l}=0, \quad s_{t} \neq d_{t} \\
& \sum_{r_{t}^{\prime} \in R, o \in P_{O}^{n}, l \in \Omega} W_{r_{l}^{\prime}, i, o}^{n, k, l} \leq 1, \quad i \in I P L_{o e o}^{n}, k \in \Omega \\
& \sum_{r_{l}^{\prime} \in R, i \in P_{l}^{n}, k \in \Omega} W_{r_{l}^{\prime}, i, o}^{n, k, l} \leq 1, \quad o \in O P L_{o o o}^{n}, l \in \Omega \\
& W B_{i, o}^{n, p}+\sum_{r_{i}^{\prime} \in R_{h^{\prime r}, l \in \Omega} l \neq k} W_{n_{l}^{\prime}, i, o}^{n, k, l} \leq 1, \quad k \in p, i \in O E P_{o o o}^{n}, o \in E O P_{o o o}^{n}
\end{aligned}
$$

$W B_{i, o}^{n, p}+\sum_{r_{i}^{\prime} \in R_{n}^{i n}, l \in \Omega, l=k} W_{r_{1}^{\prime}, i, o}^{n, k, l} \leq 1, \quad k \in p, i \in O E P_{o o o}^{n}, o \in D L_{o o o}^{n}$

In Eq. (1), we assume that the cost of one TP is equal to one unit, namely $\alpha=1$, and the energy consumed by each OEO port is equal to one Watt (W), namely $\gamma=1$.

The ILP formulation presented above is very complex. Although solving the ILP problem is still possible for some small-sized networks, it is not practical for the real-world and large-sized networks. The number of variables in an ILP formulation directly influence the computation time required solving the formulation. For example, we assume that $P=5$, $|V|=10,\left|I T P_{o o o}^{n}\right|=5,\left|O T P_{o o o}^{n}\right|=5,|R|=10,\left|I P L_{o o o}^{n}\right|=10$, $\left|O P L_{o o o}^{n}\right|=10$ and $|\Omega|=10$, then the ILP problem has $1,001,250$ variables. It is obvious that the number of variables is very large and cannot be solved in acceptable time for practical networks. Therefore, we developed two efficient heuristics CEEEM and CESPM.

\section{HEURISTIC APPROACHES}

\section{A. Network model}

The physical topology is denoted as $G_{P}(N, L, B, W, G)$, where $N, L, B, W$, and $G$ represent the set of nodes each of which is a hybrid hierarchical optical node, the set of links each of which is bidirectional and contains two unidirectional fibers with contrary direction, the set of available wavebands per fiber link, the set of available wavelengths per fiber link the waveband granularity.

\section{B. Energy consumption dynamically in OEO switch}

From the structure of hybrid hierarchical OXC node, we can see that OEO part of the node is commonly used when the request is added/dropped locally or the request is switched from one waveband to another different one, which consumes energy of APs, DPs, OEPs and EOPs. We now summarize these dynamical actions mentioned above. 
Action 1: Adding one request locally at node $n$ : This action needs one AP and one EOP, as shown in Fig.2 (a). Then this action consumes 2 (W) energy for each locally-added request at node $n$.

Action 2: Dropping one request locally at node $n$ : If drop the request using wavelength $\lambda_{i}$ on one waveband with holding $K(1 \leq K \leq G)$ requests currently, all the wavelengths in that waveband are de-multiplexed into OEO switch through $K$ OEPs, and then the wavelength $\lambda_{i}$ is dropped through one DP and residual $(K-1)$ through requests are re-multiplexed into one waveband through $(K-1)$ EOPs, as shown in Fig. 2 (b). Therefore, this action consumes $2 K(\mathrm{~W})$ energy for each locally-dropped request at node $n$.

Action 3: Switching one request from one waveband to another different one at the immediate node $n$ : If switch the request using wavelength $\lambda_{i}$ from waveband1 with holding $K(1 \leq K \leq G)$ requests currently to waveband2, all of the wavelengths used in waveband 1 are de-multiplexed into OEO switch through $K$ OEPs, then $M(0 \leq M \leq K-1)$ requests terminated at this node are directly dropped through $M$ DPs while the wavelength $\lambda_{i}$ and other $(K-1-M)$ wavelengths need to be converted into another ones on waveband2, and then these wavelengths are re-multiplexed into waveband2 through $(K-M)$ EOPs, as shown in Fig. 2 (c). Therefore, this action consumes $2 K(\mathrm{~W})$ energy for each wavelengthconvertible request at node $n$ connecting with two different wavebands.

\section{Algorithm description}

Based on the Integrated Auxiliary Graph (IAG) that includes one Virtual Topology Layer (VTL) and $|B|$ Waveband-Plane Layer (BPL) [9], two heuristics, CEEEM and CESPM are applied to jointly analyzing the port-cost and energy-consumption in hybrid hierarchical optical network. Specially, followed by EEM strategy, the first one is to find a single-hop virtual-route whose capacity is sufficient to accommodate the request on VTL or the best waveband-route on one BPL. But the disadvantage is that it only can perform the same source-destination waveband grouping. To overcome the above drawback, the second approach also allows requests between different node pairs to share capacity on common virtual-routes on entire IAG.

We consider the time complexity of routing computation in two heuristics for each request in the worst case. The time complexity of two heuristics is mainly dependent on the running times of Dijkstra's algorithm whose time complexity is $O\left(|N|^{2}\right)$. We will run one time of Dijkstra's algorithm on $\mathrm{VTL}$, and the node number of VTL is $|N|$. Then, we will run at most $|W|$ times of Dijkstra's algorithm on each BPL where the node number is also $|N|$ to compute the best waveband-route. Finally, we will run one time of Dijkstra's algorithm on IAG where the node number is $(|W|+1) \times|N|$.
Therefore, for each request in the worst case, the time complexities of two heuristics are $O\left[|N|^{2}+|W| \cdot|N|^{2}\right]$ and $O\left[|N|^{2}+|W| \cdot|N|^{2}+(|W|+1)^{2} \cdot|N|^{2}\right]$, respectively,

\section{SIMULATION AND ANALYSIS}

We simulate a dynamic network environment with the assumptions that connection requests arrive according to an independent Poisson process with arrival rate $\beta$ and that the connections' holding times are negatively exponentially distributed $1 / \mu$, i.e., the Network Load (NL) is $\beta / \mu$ Erlang. In simulation, we set $\mu$ to 1 . The test network is NSFNET in Fig. 3, where each node is assumed to be equipped with 4 transceivers, each link has 2 waveband tunnels and each waveband tunnel at most provides $G$ units of wavelength capacity-level bandwidth, each of which is equal to the required bandwidth of each connection request. The computer in simulation is configured with $1 \mathrm{G}$ DDR RAM and $2 \mathrm{GHz}$ $\mathrm{CPU}$, and the software is $\mathrm{VC}++6.0$. We compare CESPM algorithm to CEEEM algorithm on the performance of Mean Blocking Probability (MBP), Average Port Cost (APC) and Average Consumed Energy (ACE). The MBP is defined as the ratio of the total blocked requests to the total arrival requests, APC is defined as the average cost of Transmitting Ports (TPs) consumed by each accepted connection request and $\mathrm{ACE}$ is defined as the average energy consumed by OEO part of the node for each accepted connection request. In simulation, we take different values of NL. All results are average by simulating $10^{4}$ connection requests.

In Fig.4, we can see that the MBP in CESPM is lower than that in CEEEM, which means that the mean blocking probability in CESPM is better than that in CEEEM. The reason for this is that, in CEEEM, when a single-hop waveband tunnel is failed to be found, the connection request will be blocked, while in CESPM, that connection request can be switched into a multi-hop paths with a series of waveband tunnels, such that the mean blocking probability in CESPM is better. We can also see that, when the WG increases, the MBP in CESPM gradually becomes small, which means that the mean blocking probability in CESPM is gradually improved when the WG increases. The reason for this is that, when the WG increases, there will be more connection request can be merged into waveband tunnel(s), such that the mean blocking probability is improved.

In Fig.5, we test the performance of APC for CESPM and CEEEM. It is shown that the APC in CESPM is smaller than that in CEEEM, which means that the average cost of TPs consumed by each accepted connection request in CESPM is lower than that in CEEEM. The reason for this is that, connection requests can only be merged into single-hop waveband tunnel followed by End to End Merging strategy (EEM) in CEEEM, and if such single-hop waveband tunnel is not found, these connection requests will be switched respectively, which consumes a large number of TPs, while in 


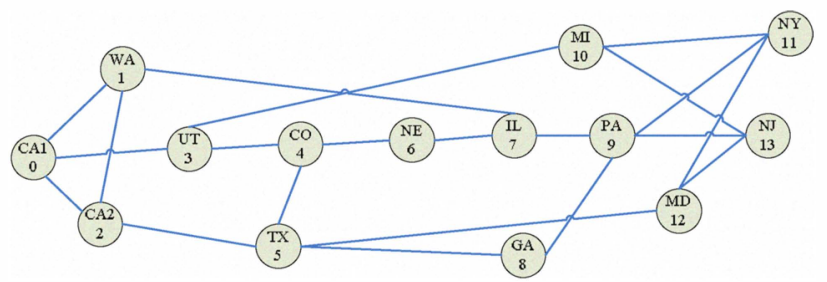

Fig.3 Simulation Topology: NSFNET backbone network topology, where each line represents a bidirectional fiber link

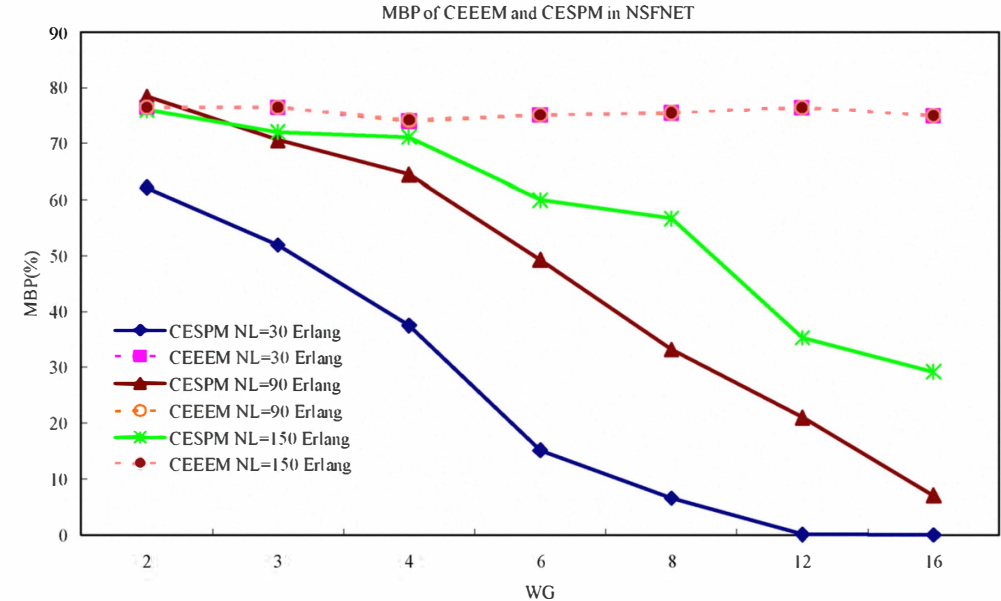

Fig.4 Comparison of MBP between CEEEM and CESPM in different network load APC of CEEEM and CESPM in NSFNET

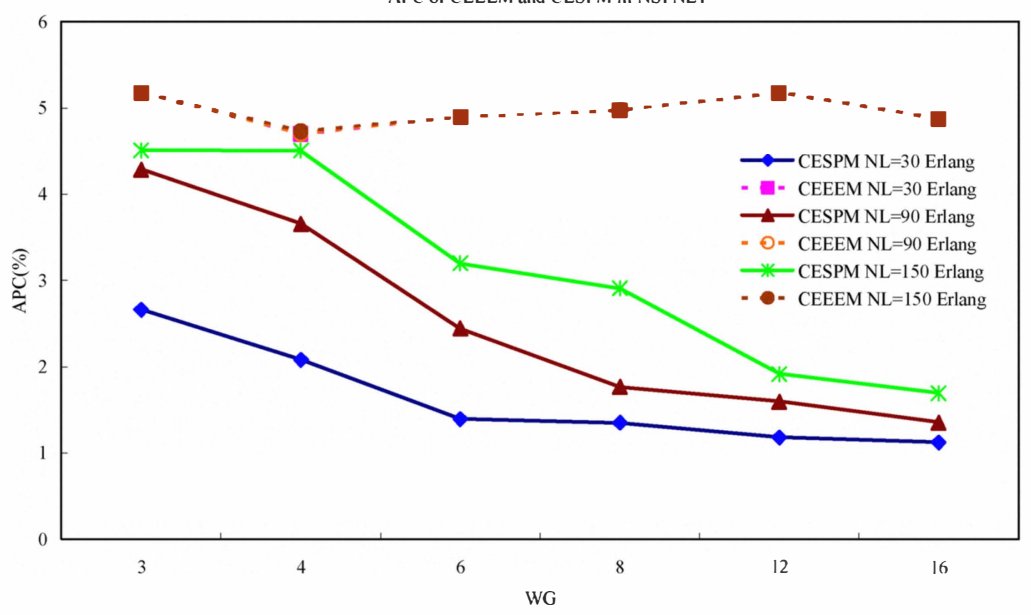

Fig.5 Comparison of APC between CEEEM and CESPM in different network load

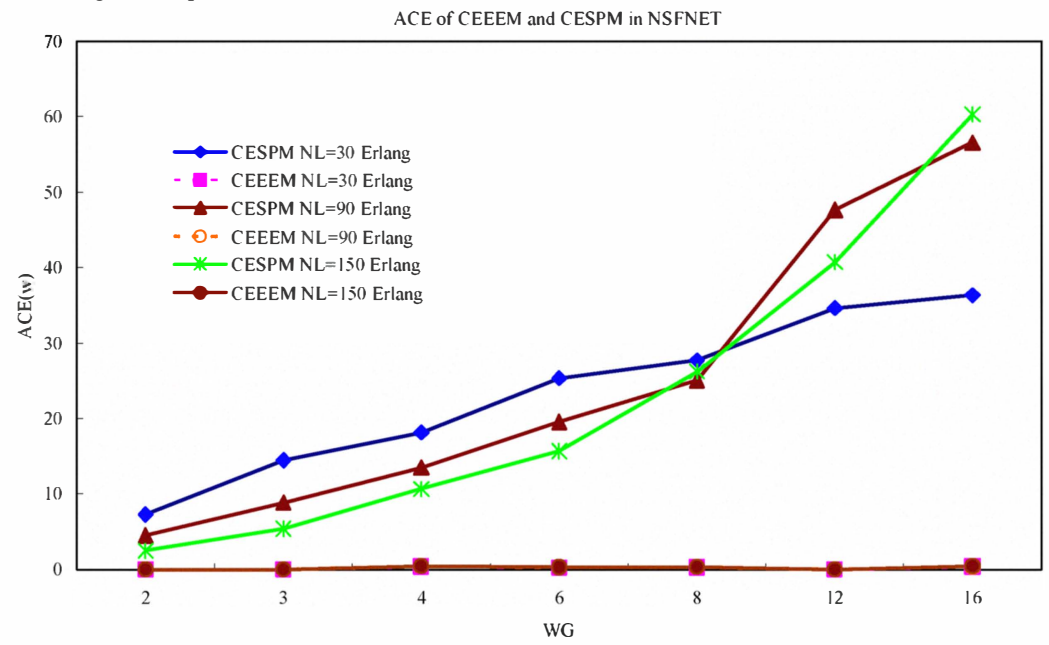

Fig.6 Comparison of ACE between CEEEM and CESPM in different network load 
CESPM, followed by Sub Path Merging strategy (SPM), connection requests can also be merged into a series of waveband tunnels instead of switching these requests respectively, such that the average cost of TPs consumed by each accepted connection request in CESPM is lower. We can also see that when the WG increases, the APC of CESPM gradually becomes small, which means that the average cost of TPs consumed by each accepted connection request in CESPM is further saved when the WG increases. The reason for this is that, when the WG increases, there will be more connection request can be merged into waveband tunnel(s) instead of consuming a large number of TPs used to switch these request respectively, such that the average cost of TPs consumed by each accepted connection request is saved.

In Fig.6, we test the ACE for CESPM and CEEEM. It is shown that compared to CEEEM, the values of energy consumed by OEO part of the node for each accepted request in CESPM are increased. The reason for this is that, compared to CEEEM, there will be more connection requests can be merged into waveband tunnel(s), which consumed more energy of OEO part to add/drop these connection requests and perform wavelength conversions when two adjacent waveband tunnels consume different wavebands, such that the average energy consumed by OEO part of the node for each accepted connection request is increased. We can also see that when the WG increases, the ACE in CESPM gradually becomes big, which means that the energy consumed by OEO part of the node becomes high when the WG increases. The reason for this is that, when the WG increases, there will be more connection requests will be merged into waveband tunnels, and then more APs, DPs, OEPs and EOPs in OEO part of the node will be used, such that the more corresponding energy is consumed.

From the results and analysis in Fig. 4, 5 and 6, it is shown that, the performance of APC is contrary to the performance of ACE; compared to CEEEM, CESPM has the better performance of APC but the performance improvement is achieved at the expense of higher energy consumption in OEO part of the node; compared to CESPM, CEEEM has the better performance of $\mathrm{ACE}$ but the performance improvement is achieved at the expense of higher cost of used ports.

\section{CONCLUSION}

In this paper, we devised a new routing method for hybrid hierarchical OXC node and develop a port-cost and energyconsumption minimization model for hybrid hierarchical optical network. The optimal ILP solution was for networks of smaller size only. For larger networks, we used heuristic CEEEM and CESPM algorithms. Simulation results of two heuristic approaches show that, the cost of used ports is contrary to the energy consumed by OEO switch. In our future study, in order to obtain low blocking probability and satisfactory trade-off performance, we plan to design a new adaptive algorithm that can choose a rational waveband merging strategy dynamically according to the current performance of the port-cost and energy-consumption in the whole network.

\section{ACKNOWLEDGMENT}

This work was supported in part by the Fundamental Research Funds for the Central Universities (N090604007, N090504001, N090504003, N090504006, N090404014), the National Natural Science Foundation of China (60802023, 70931001), the Fok Ying Tung Education Foundation (121065), the National Science and Technology Pillar Program (2008BAH37B03, 2008BAH37B07), the National High-Tech Research and Development Plan of China (2007AA041201), the Program for New Century Excellent Talents in University (08-0095), and the Specialized Research Fund for the Doctoral Program of Higher Education (20070145096, 20070145017).

\section{REFERENCES}

[1] X. Cao, V. Anand, C. Qiao: Framework for waveband switching in multigranular optical networks: part I-multigranular crossconnect architectures, Journal of Optical Networking, 2006, vol.5, no.17, pp: 1043-1055.

[2] X. Cao, V. Anand, C. Qiao: Framework for waveband switching in multigranular optical networks: part II-wavelength/waveband conversion and survivability, Journal of Optical Networking, 2006, vol.6, no.1, pp: 48-62.

[3] J. Baliga, R. Ayre, K. Hinton, R. Tucker: Photonic Switching and the Energy Bottleneck, Photonics in Switching, 2007, pp:125-126.

[4] J. Baliga, R. Ayre, K. Hinton, W. V. Sorin. Energy Consumption in Optical IP Networks, Journal of Lightwave Technology, 2009, vol.27, no. 13, pp: 2391-2403.

[5] G. Shen, R. Tucker. Energy-minimized design for IP over WDM networks, IEEE/OSA Journal of Optical Communications and Networking, 2009, vol. 1, no. 1, pp. 176-186.

[6] R. Izmailov, S. Ganguly, T. Wang, Y. Suemura, Y. Maeno, S. Araki: Hybrid hierarchical optical networks, IEEE Communications Magazine, 2002, vol.40, no.11, pp: 88-94.

[7] S. Ganguly, N. Tu, R. Izmailov, H. Mouftah: Waveband routing and merging in hybrid optical networks, ICC, 2004, vol.3, pp:1615-1621.

[8] M. Li, W. Yao, B. Ramamurthy: Same-destination-intermediate grouping vs. end-to-end grouping for waveband switching in WDM mesh networks, ICC, 2005, vol.3, pp: 1807-1812.

[9] L. Guo, X. Wang, W. Ji, W. Hou, H. Li, H. Wang, X. Liu: A novel waveband switching routing algorithm based on integrated auxiliary graph in WDM optical networks, ICCT, 2008, pp:383-386. 